\title{
Ferro-resonance Overvoltage Identification Using Earth Capacitance and Excitation Inductance of Ratio Method
}

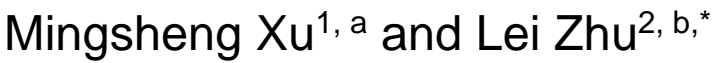 \\ ${ }^{1}$ State Grid Huai'an Power Supply Company, Huai'an, 223002, China \\ ${ }^{2}$ School of Electrical Engineering, Southeast University, Nanjing, 210096, China \\ ajiayouzhulei@163.com, b705773474@qq.com
}

Keywords: ferromagnetic resonance, EMTP, ratio method, overvoltage identification.

\begin{abstract}
The ferromagnetic resonance overvoltage of power system often occurs for a long time, which seriously endangers the safe operation of electrical equipment and power grid. The feature extraction and recognition of ferromagnetic resonance overvoltage is helpful for taking targeted measures to restrain overvoltage quickly, and it is of great significance to guarantee electric power system running in reliable and steady status. Based on the analysing the over-voltage and over-current waveform characteristics of different resonance types by EMTP simulation software, a method of ferromagnetic resonance overvoltage recognition is proposed which is named earth capacitance and excitation inductance ratio. The theoretical foundation of this method is visualized, and a lot of actual data show that this method is feasible and effective in ferromagnetic resonance overvoltage recognition.
\end{abstract}

\section{Introduction}

There are many inductive and capacitive components in the power system. When the system operation or failure occurs, these component parameters will change. Some of the inductance and capacitance components of the circuit may form a resonance, causing resonance over-voltage. In the neutral non-earthed system, the bus voltage on the electromagnetic voltage transformer (referred to as PT) usually need to ground the neutral point. When the line break, no-load bus closed or single-phase ground fault disappears and so on, the non-linearity of the magnetizing inductance of the PT inductor makes the iron core saturate, matching with the system's capacitance to ground capacitance, causing the ferromagnetic resonance overvoltage. This is the most common over-voltage accident in the power grid, having a serious impact on the safe operation of power systems ${ }^{[1-4]}$.

When the system occurs over-voltage accident, the over-voltage type is needed to be determined so that targeted measures can be taken to suppress over-voltage. Ferromagnetic resonance is extremely sensitive to changes in system parameters, and it contains frequency, fundamental frequency and high frequency ferromagnetic resonance. Some measures are effective to eliminate a frequency resonance, but on a different resonance frequencies may be useless. However, the existing over-voltage monitoring system does not yet have the ability to analyse and identify, the type of over-voltage needs to be manually determined. Unfortunately, it is easy to misjudgment and not conducive to the analysis and solve accidents timely. Therefore, it is very important to study the characteristics of ferromagnetic resonance over-voltage feature extraction and type recognition, which is of great significant for the rapid suppression of over-voltage $\mathrm{e}^{[5-6]}$.

In the actual power system, the frequency of ferromagnetic resonance is most likely to occur, followed by the fundamental frequency ferromagnetic resonance, high frequency ferromagnetic resonance generally does not appear. In this paper, the ferromagnetic resonance overvoltage is classified into the ferromagnetic resonance and fundamental frequency ferromagnetic resonance according to their respective phenomena and characteristics. The parameters of the ground capacitance will be changed to simulate different types of ferromagnetic resonance when the fault occurs, and ATP-EMTP will be used to verity. Combining with the experimental results and the actual engineering application, it is considered that the ratio of the capacitive reactance value to the 
inductive reactance value of the PT magnetizing inductor affects the type of ferromagnetic resonance. The conclusion is facilitate for staff members to determine the type of ferromagnetic resonance quickly, and prevent further expansion of the accident.

\section{The Mechanism of Ferromagnetic Resonance}

As the Fig.1 shows, this is a typical system equivalent circuit. $E_{A} 、 E_{B} 、 E_{C}$ are three-phase power electromotive forces, $L_{A} 、 L_{B} 、 L_{C}$ are three-phase inductances, and $C_{0}$ is the ground capacitance. $Y_{A}=\omega C_{0}-1 / \omega L_{A}$. Under normal operating conditions, $L_{A}=L_{B}=L_{C}, Y_{A}=Y_{B}=Y_{C}$. At this point the three-phase circuit is in basic balance, and the system neutral point displacement voltage is zero, $\dot{U}_{0}=\frac{\dot{E}_{A} Y_{A}+\dot{E}_{B} Y_{B}+\dot{E}_{C} Y_{C}}{Y_{A}+Y_{B}+Y_{C}}$.

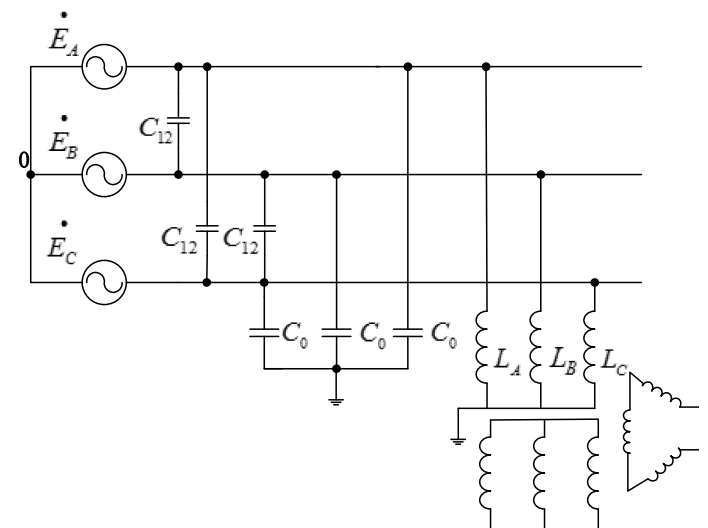

Figure 1. Equivalent circuit diagram of ferro-resonance

When it is happened some of the impact of disturbance circuit breaker closing in the no-load bus or line single-phase instantaneous ground fault disappears, may causing one or two phase voltage transient rises. If three-phases of PT are hit by different degrees, the degree of saturation will also vary. $Y_{A} \neq Y_{B} \neq Y_{C}$, and the neutral point displacement voltage will rise. The frequency of the ferromagnetic resonance is determined by the inductance and capacitance parameters of the oscillation circuit. Due to the non-linearity of PT inductor, the inductance value is not a parameter, and waveform distortion occurs under the action of AC power. Therefore there is no fixed oscillation frequency in the loop, including higher harmonic resonance, fundamental resonance and frequency resonance. The characteristics of the three resonances are also different: (1)Frequency resonance fault has the following characteristics: a. the line voltage of three-phase increased at the same time, and usually not more than 2.5 times the phase voltage; b. the current on PT increased significantly.(2)Fundamental resonance fault has the following characteristics: a. the line voltage of two-phase rise, and the rest reduced or two phase reduction, one phase rise, and the voltage is usually not more than 3.5 times the phase voltage; $b$. the current on PT increased significantly.(3)High-frequency resonance fault has the following characteristics: a. the line voltage of three-phase increased at the same time, and usually not more than phase voltage; $b$. the current on PT is not high.

\section{The Simulation of Ferromagnetic Resonance}

\subsection{Establishment of Simulation Model}

In this paper, EMTP is used to simulate for electromagnetic transient. According to operational experience, single-phase-to-ground fault is most likely to resonate in a neutral point ungrounded system. Because of the capacitive current flow through the point of failure, the sound phase voltage increased to line voltage; when

the ground fault disappears, the sound relative to the ground voltage will return to the original phase voltage. The charge which is accumulated during the fault needs to be released, while it can 
only be released by PT neutral in the neutral point of ungrounded system, RESULTING in the saturation of PT. Then the excitation inductance drops sharply, and it may cause resonance. As shown in Figure 2, $K_{a} 、 K_{b} 、 K_{c}$ are single-phase ideal switch, controlling the closing and sub-gate of three bus respectively. Phase $\mathrm{C}$ is connected to the ground by $K_{1}$, to simulate the occurrence and disappearance of single-phase ground fault. $C_{a} 、 C_{b} 、 C_{c}$ is line-to-ground capacitance respectively. If the neutral point does not pick any electrical equipment in ATP, the simulation will be wrong, so switch $K_{0}$ will be installed to the ground and is in a state of open during the simulation.

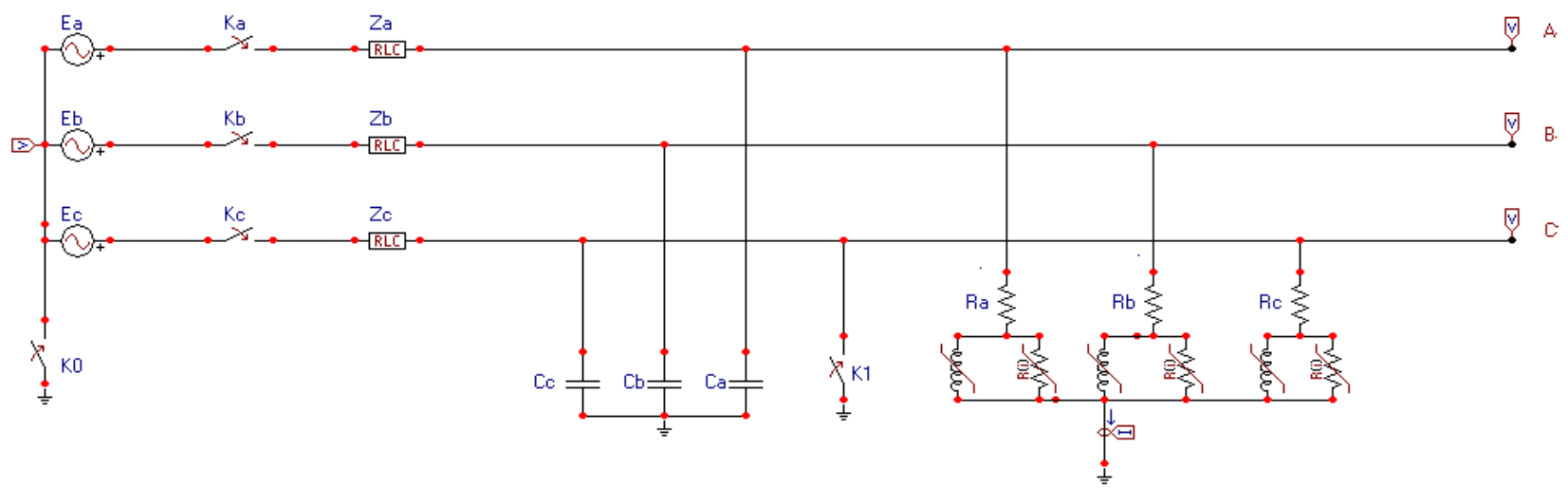

Figure 2. Equivalent simulation model of $10 \mathrm{kV}$ neutral point unearthing system

\subsection{Simulation Parameters}

It is assumed that the line parameters of three-phase are symmetrical, $E_{a} 、 E_{b} 、 E_{c}$ are operating voltages of three-phase. Setting the voltage of the system is $10 \mathrm{kV}, Z=1+j 1 \Omega$, the impedance of the line and the transformer. According to engineering experience, capacitance of line-to-ground in transmission is $0.005 \mu \mathrm{F} / \mathrm{km}$, the power supply line radius of $10 \mathrm{kV}$ distribution network is generally not more than $15 \mathrm{~km}$, setting $C_{0}=0.25 \mu \mathrm{F} . K_{a} 、 K_{b} 、 K_{c}$ are closed at $-0.5 \mathrm{~s}, K_{1}$ is closed at $0.4 \mathrm{~s}$ and opened at $0.6 \mathrm{~s}$.

\subsection{Simulation Analysis}

In this paper, by changing the value of capacitance $C_{0}$, analysis the characteristics of simulation waveform, which can be shown in Figure 4.

(1)When $C_{0}=0.25 \mu \mathrm{F}$

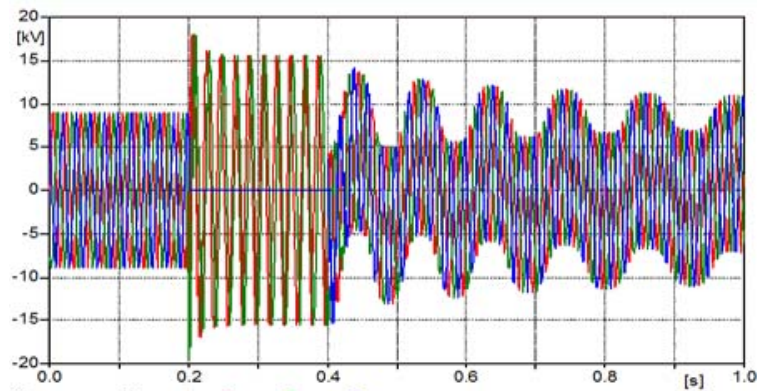

(a) Voltage waveform of three phase

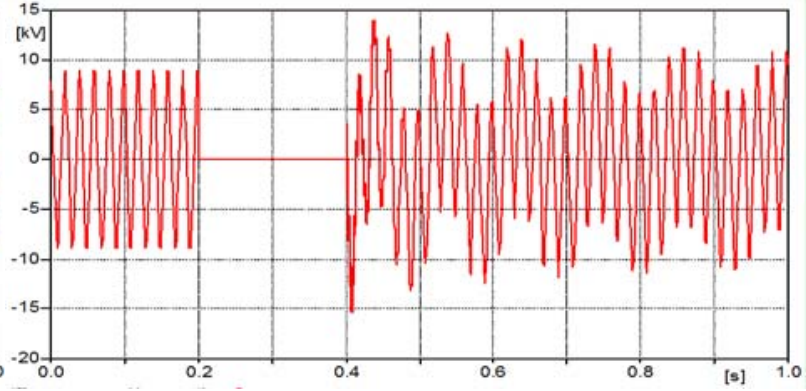

(b) voltage waveform of $\mathrm{C}$ phase

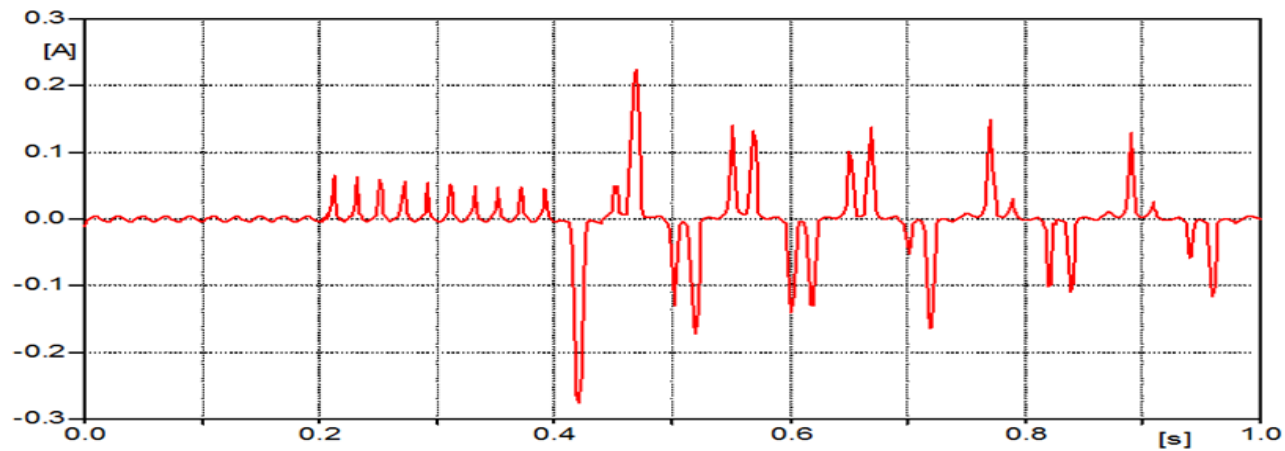

(c) Excitation current on PT 
(2) When $C_{0}=0.01 \mu \mathrm{F}$

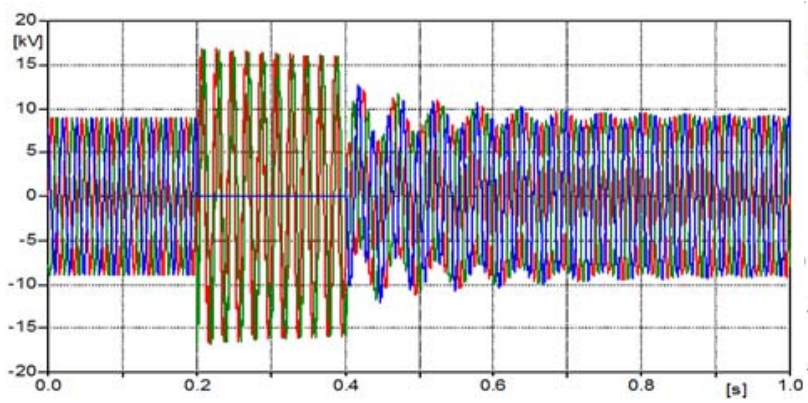

(d) Voltage waveform of three phase

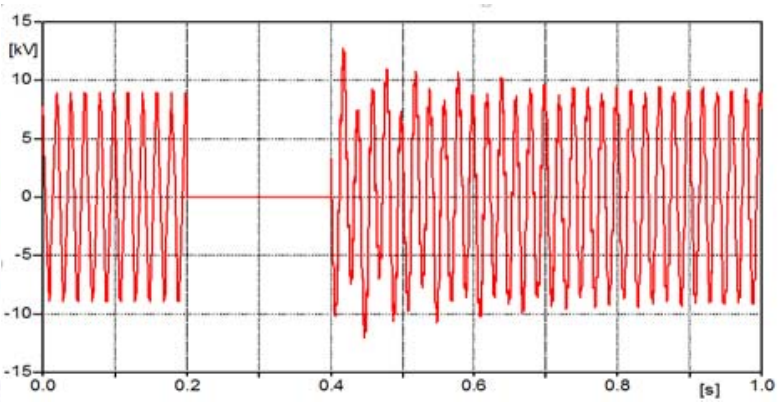

(e) voltage waveform of $\mathrm{C}$ phase

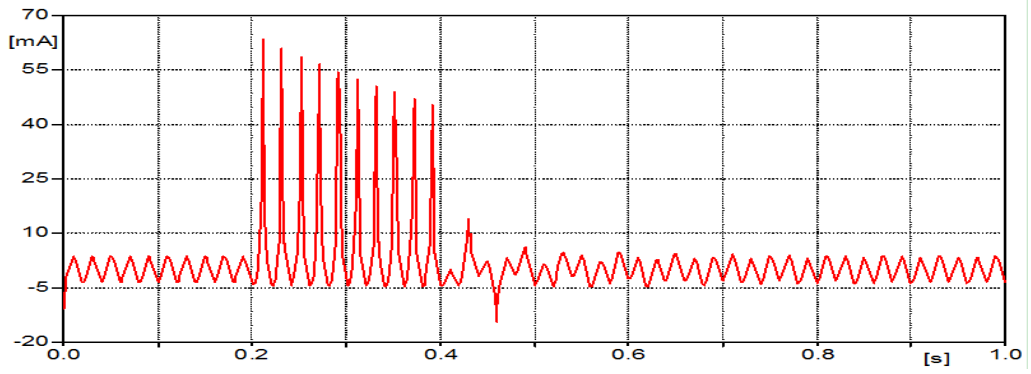

(f) excitation current on PT

Figure 4. Waveform of simulation

When $C_{0}=0.25 \mu \mathrm{F}$, according to the simulation waveform above, it can be seen that three-phase voltage increased at the same time, swing cyclically, and the over-voltage is 1.7 times the phase voltage. Excitation current on PT rise up to 30 times the rated value. So it can be judged as the frequency-division resonance. Combined with the non-linear characteristic curve of the magnetizing inductance in the voltage transformer, the reactance value $X_{m}$ and $X_{c 0} / X_{m}=0.05$ can be calculated. When $C_{0}=0.01 \mu \mathrm{F}$, it can be judged at this time the fundamental resonance occurred based on the waveform characteristics above. At this time, it can be calculated that $X_{c 0} / X_{m}=0.127$.

\section{The Identification Process of Ratio Method}

In addition to the characteristics of ferromagnetic resonance can be identified based on the characteristics of the above categories, this paper, a new method is proposed after a large number of simulation calculations-based on the capacitance and excitation inductance ratio method of ferromagnetic resonance overvoltage recognition method. When the line-to-ground capacitance parameter changes, PT inductance value will change, by dividing the interval where the location, the type of ferromagnetic resonance can be determined. As the following rule: when $X_{c 0} / X_{m} \in[0.01$, 0.07], it is frequency resonance; when $X_{c 0} / X_{m} \in[0.07,0.55]$, it is fundamental resonance; when $X_{c 0} / X_{m} \in[0.55,2.8]$, it is high-frequency resonance and other values are considered not ferromagnetic resonance. In summary, the conclusion obtained by the ratio method is consistent with the waveform analysis, which proves the reliability and practicability of the criterion. Under normal circumstances, high-frequency resonance is not easy to occur, so this article does not do simulation analysis.

\section{Conclusion}

In this paper, the classification of ferromagnetic resonance overvoltage is verified by the waveform characteristics firstly, and then a new classification method of ferromagnetic resonance overvoltage classification is proposed based on the ratio of capacitance to inductance. By changing the value of ground capacitance, the frequency of ferromagnetic resonance and fundamental frequency ferromagnetic resonance were simulated respectively, the results of the new method is 
consistent with waveform analysis, it is concluded that ferromagnetic resonance over-voltage can be distinguished with the ratio method effectively.

\section{References}

[1]. DU Lin, LI Xin, WU GAO Lin, DENG Bang-fei. Ferro-resonance Overvoltage Identification Using Three Feature Parameters of Ratio Method [J].High Voltage Engineering, 2011, 09:2241-2249.

[2]. LIANG Zhi rui, DONG Wei, LIU Wen-xuan, HUANG Jia-zhen, WU Qun-xiong. Simulation Analysis on Ferroresonance of Potential Transformer [J].High Voltage Apparatus, 2012, 11:18-23.

[3]. GE Dong, LU Tie-cheng, WANG Ping .Study on simulation calculation of ferro-resonance eliminat- ion in power distribution system[ J] .High Voltage Engineering, 2003 , 29(11):15-17 .

[4]. ZHANG Bo, LU Tie cheng, DU Xiao-lei. Analysis of harmonic balance based fundamental resonance of the ferro-resonance circuit in power system [J] .High Voltage Engineering, 2006, 32(1):94-96.

[5]. ZHOU Jie-lian, WU Shao-zhen. The effective measures to prevent from ferro-resonant overvol tage of voltage transformer [J] .High Voltage Engineering, 2001, 27(4):53-55 .

[6]. ZHANG Ping. The limited method on resonanc overvoltage of power grid [J]. Shan xi Coking Coal Technology, 2009(3):28-30. 